Metropolitan-Vickers Electrical Co., Ltd., follows closely Dr. Bush's original machine at the Massachusetts Institute of Technology, but several modifications in detail have been made.

The machine is being built in two sections, one of which is now complete. This completed section comprises four integrators and two input tables, the output table, and a special camera designed and built by Messrs. Newman and Guardia, Ltd., for the photographic recorder. One of the input tables is of special construction, for handling equations describing the behaviour of a system in which the rate of change of a quantity at time $t$ may depend explicitly on its value, or on the values of other quantities, at time $t-T$ (where the time-lag $T$ may be constant or variable) as well as on their values at time $t$. The second section, now under construction, will comprise four further integrators, making eight altogether, and probably four further input tables, and, as explained above, the greater range will very greatly increase the value of the machine.
The construction of this differential analyser has been made possible, first through the great generosity of Mr. Robert McDougall, deputy treasurer of the University, who first gave to the University a donation to cover the estimated cost of the first section, now completed, and has recently supplemented this by a further donation to cover the completion of the machine in accordance with the original estimates; secondly, by the friendly and generous co-operation of Dr. Bush himself, who freely gave his drawings, and several suggestions for improvement, and helped greatly by his advice based on experience of construction and operation of his own machine; and thirdly, through the co-operation of Mr. A. P. M. Fleming, of Metropolitan-Vickers Electrical Co., Ltd., who undertook the construction of the machine, and of those members of the firm who have been concerned in its design, construction and erection.

\footnotetext{
1 V. Bush, J. Franklin Inst., Oct. 1931, and "Proc. Internat. Congress on Applied Mechanics", Cambridge, 1934.

2 Sir W. Thomson, Proc. Roy. Soc., 24, $269 ; 1876$. NATURe, 135, 535, April 6, 1935.
}

\title{
Royal Society Discussion on Supraconductivity
}

$\mathrm{I}^{\mathrm{N}}$ opening the Royal Society discussion on supraconductivity, Prof. J. C. McLennan referred first to new methods of helium liquefaction which have recently been developed. In Prof. F. Simon's method, high pressure helium gas is cooled to liquid hydrogen temperatures and then allowed to expand through a valve, a small quantity of liquid helium being produced which is quite adequate for many types of experiments. A second method, developed by Prof. P. Kapitza at Cambridge, applies to helium the method first used by Claude for the liquefaction of air, part of the gas doing external work in an expansion engine and so cooling the remainder below the inversion temperature of the Joule-Thomson effect, whence it can be liquefied by expansion.

Prof. McLennan then discussed the rapid progress in the attainment of temperatures near the absolute zero made possible by the adiabatic demagnetisation method, the latest experiments of de Haas with potassium chromium alum having reached a temperature of $0.0044^{\circ}$ on the scale obtained by measuring the magnetisation and using an extrapolated Curie Law to obtain the temperature. Progress is at the same time being made in the establishment of the thermodynamic scale in this temperature region. Prof. W. H. Keesom reported that the thermodynamic scale has been established down to $0.9^{\circ} \mathrm{K}$. using the helium thermometer. Prof. Simon reported the results of an ingenious method developed at
Oxford in which the heat, $d Q$, required to warm up the salt from its lowest temperatures to its initial temperature is determined by using $\gamma$-rays to warm up the salt. Since the entropy change, $d S$, on magnetisation can be calculated and the cooling is adiabatic, the temperature on the absolute scale is obtained from $d Q / d S$. The results show that the thermodynamic scale and the magnetic scale using iron ammonium sulphate do not differ by more than 10 per cent down to $0.08^{\circ} \mathrm{K}$., but that below this temperature the thermodynamic temperature is greater than the magnetic temperature. For these lower temperatures the shape of the specimen has a large effect on the magnetic temperature owing to the increasing importance of the demagnetising coefficient.

Prof. McLennan referred also to the prediction that at these low temperatures the effect of nuclear magnetic moments should become important, owing to the thermal energy becoming comparable with the energy of magnetisation due to nuclear moments. Prof. Simon considers that it may be possible to use a two-stage demagnetisation process for the attainment of the lowest temperatures, the nuclear moments becoming effective in the second stage. Dr. Heitler's prediction in the discussion that it would take a year for equilibrium to be set up owing to the smallness of the nuclear interaction failed to shake Prof. Simon's determination to try the experiment.

The production of these low temperatures has 
extended the list of supraconducting metals, and Prof. McLennan gave a list of fifteen such metals with transition temperatures ranging from $0 \cdot 40^{\circ} \mathrm{K}$. to $9 \cdot 2^{\circ} \mathrm{K}$. As Mr. J. D. Bernal pointed out in the discussion, these metals lie in Groups IVa, Va, VIa and IIb, IIIb, IVb of the Periodic Table, and in no others. A number of supraconducting alloys, on the other hand, have been discovered, such as the gold-bismuth series, the components of which lie on either side of these groups and are not themselves supraconducting. Mr. Bernal considers that supraconductivity has little to do with the crystal lattice, since supraconductors of many crystal types are found. Prof. Keesom pointed out, however, that one of the varieties of tin is supraconducting whilst the other is not.

Prof. McLennan referred next to the remarkable phenomenon of the 'Meissner effect'. When a metal sphere placed in a weak magnetic field is cooled down to the temperature at which it becomes supraconducting, the lines of magnetic induction move out of the sphere at the transition temperature, and the field outside takes precisely the form which would be expected if $B=0$ in the interior of the sphere, supraconductivity being thus characterised by the conditions $B=0, E=0$. When the tangential component of the magnetic field reaches the critical value for supraconductivity, the lines of force re-enter and supraconductivity disappears.

Dr. Meissner described a number of further experiments on this phenomenon. Measurements have been made of the magnetic field in the space round a supraconducting cylinder placed with its axis perpendicular to the field. The lines of force in the hollow of the cylinder do not move out, but on the contrary are increased in density when the cylinder becomes supraconducting, and on removal of the external field these lines of induction remain almost unchanged, thus imparting a magnetic moment to the cylinder. As the external field is increased, the normal component of the field outside the cylinder increases, showing increasing penetration of the lines of force.

Dr. K. Mendelssohn described experiments on this phenomenon which show that only in the ideal case of a perfectly pure metal and a perfect lattice do all the lines of induction disappear from the supraconducting sphere. When the metal is impure, some of the lines of induction appear to be 'frozen in', a rod of moderately pure lead retaining about ten per cent of the flux. The results can be explained, he considers, by the formation of annular supraconducting regions in which the flux is 'locked', the formation of such regions being aided by the effect of impurities in increas. ing the local transition temperature for supraconductivity.
Since a state of zero magnetic induction is equivalent to a diamagnetic susceptibility of $-\frac{1}{4} \pi$, experiments on the force exerted by an inhomogeneous magnetic field on a lead sphere should show an enormous increase in the pull when supraconductivity sets in. An experiment of this type carried out at Cambridge was described by $\mathrm{Mr}$. Shoenberg, the force for weak fields corresponding precisely to that expected. As the field is increased, the susceptibility remains constant until the field at the equator reaches that critical for supraconductivity. At this point, the lines of force begin to penetrate the sphere, and the susceptibility decreases to zero as the main part of the external field reaches the critical value.

Considerable discussion took place on the evidence provided by low temperature phenomena on the behaviour of electrons in metals. Thus, for supraconducting metals of high purity, the thermal conductivity is increased when the supraconductivity is destroyed by a magnetic field, in agreement with the hypothesis that the supraconducting electrons are an appreciable fraction of the ordinary conduction electrons and that they become available for heat transfer when supraconductivity is destroyed. On the other hand, the thermal resistance should tend to zero near absolute zero, whereas the actual resistivity shows a minimum and begins to rise again at the lowest temperatures, one striking case being reported by Prof. Simon in which the thermal resistivity of a copper rod became equal to that of glass at room temperature.

Prof. Keesom described experiments on the specific heats of metals which show the presence of an additional heat capacity due to the electrons, increasing linearly with temperature in the case of tin up to $9^{\circ} \mathrm{K}$. Prof. N. F. Mott considers that additional evidence for the heat capacity due to electrons is provided by the specific heat of metals such as nickel at temperatures of $1000^{\circ} \mathrm{K}$., where $C_{v}$ is greater than the Debye value of $3 R$ by at least $1 R$, the maximum contribution for electrons being $3 / 2 R$.

Further experiments on the effect of electrons in metals were demanded by Prof. R. de L. Kronig, who suggested that the reflecting power of metals should show measurable changes on transition from the supraconducting to the conducting state. Changes should also occur in the fine structure of $\mathrm{X}$-ray absorption edges and in the transmission of wireless waves by thin metal films.

Prof. L. Brillouin and Dr. F. London discussed the state of the theories of supraconductivity. The former emphasised the facts which a theory has to explain, and referred to a proof by Bloch that no classical theory can explain a stable state of persistent currents. Dr. London opened a new 
attack on the problem by refusing to consider supraconductivity as the limiting case of ordinary conductivity and by considering it as a more elementary state in which the whole metal behaves like a large diamagnetic atom. A new equation, $\lambda C$ curl $\boldsymbol{J}=-H$, relating current density and magnetic field, is postulated, this assumption replacing Ohm's Law in a supraconductor and leading directly to a solution in which stable persistent currents are possible, these currents being confined to a depth of $10^{-5} \mathrm{~cm}$. below the surface of the conductor.

\section{Obituary}

Prof. H. M. Macdonald, o.B.E., F.r.s.

$\mathrm{H}$ ECTOR MUNRO MACDONALD was born in 1865 at Fearn, Ross-shire, and educated at Tain Academy, Aberdeen Grammar School and the University of Aberdeen, proceeding in 1886 to Clare College, Cambridge, and taking the Mathematical Tripos in 1889. The list of Wranglers was one of considerable distinction : Sir Gilbert Walker was senior, Sir Frank Dyson second, Macdonald fourth and A. S. Ramsey (president of Magdalene) sixth. He was soon elected to a fellowship at Clare, and in 1891 was Smith's Prizeman.

During Macdonald's formative period, the professorial chairs at Cambridge were occupied by Stokes, Adams (the discoverer of Neptune), Cayley, J. J. Thomson and G. Darwin ; while of the other teachers, he seems to have owed most to Routh, Hobson, Glazebrook and Larmor. But perhaps his greatest debt was to Verdet's "Leçons d'optique physique", Maxwell's "Electricity and Magnetism" and the works of Horace Lamb and the third Lord Rayleigh.

Macdonald's first published papers were on hydrodynamics and the mathematical theory of electricity -waves in canals, electrical distributions on conductors of various shapes, and self-induction. In 1897 , however, he began to write on pure mathematies - the relation between convergent series and asymptotic expansions, the zeros and the addition theorem of Bessel functions, various Bessel integrals, spherical harmonics and Fourier series. In the paper on the zeros of the Bessel functions (Proc. Lond. Math. Soc., 29 ; 1898), he gave the result since known as Macdonald's theorem, that the number of zeros of a function $f(z)$ in the region bounded by a contour at each point of which $|f(z)|=$ a constant, exceeds the number of zeros of the derived function $f^{\prime}(z)$ in the same region by unity, the function $f(z)$ being supposed analytic in the region.

Macdonald's reputation as a discoverer was, however, chiefly due to a third group of researches, which began with his Adams Prize essay of 1902 on electric waves, and was continued in a paper of 1903 on the bending of electric waves round a conducting obstacle, two memoirs on the diffraction of electric waves round obstacles (Phil. Trans. Roy. Soc., 1911-12), several papers on the diffraction of light by opaque prisms, straight edges, etc. (Proc. Lond. Math. Soc., 1913-15), and a series of papers (Proc. Roy. Soc., A) from 1914 onwards on the transmission of electric waves round the earth's surface.

In 1905 Macdonald left Cambridge on being appointed to the chair of mathematics in his old
University of Aberdeen. The value of his work was recognised by the fellowship of the Royal Society in 1901, an honorary fellowship of Clare in 1914, a Royal Medal of the Royal Society in 1916, the presidency of the London Mathematical Society in 1916-18, and the Honorary LL.D. of Glasgow in 1934. During the last thirty years of his life, he took an active part in the administrative work of the University of Aberdeen and of educational institutions in the north-east of Scotland, and was almost invariably one of the delegates appointed to any conference of representatives of the four Scottish Universities. In recognition of his scientific eminence and public services, a subscription portrait was presented to the University of Aberdeen in 1933.

Macdonald never married. $\mathrm{He}_{\mathrm{e}}$ died after a short illness on May 16, $1935 . \quad$ E. T. WhITTAkER.

Prof. W. R. Hodgknnson, c.B.E.

ON April 8, at Blackheath, died, at eighty-three years of age, Prof. William Richard Hodgkinson, one of the older school of chemists, whose interests in chemistry covered a wide field. Born at Sheffield in 1851, and educated at the Royal Grammar School there, he early came under the influence of Sorby, the father of metallography, and to this is to be traced his later work on metals and their treatment.

Having gained a scholarship to the Royal School of Chemistry and of Mines in London, Hodgkinson studied geology, and under Sir Edward Frankland organic chemistry, before proceeding to the University of Würzburg, where his natural feeling for research was stimulated by Prof. Wislicenus, whose textbook on "Organic Chemistry" he translated. On returning to England his investigations at the Royal College of Science were mostly in organic chemistry, and in this branch of chemistry he published papers on such subjects as the action of the alkali metals on organic bodies, with W. H. Perkin, junr., and on organic bases and on naphthalene derivatives, with Dr. W. Limpach, whose sister he married nearly fifty years ago.

After a short period at the Royal Military Academy, Hodgkinson became lecturer in chemistry and metallurgy at the Ordnance College, and later professor of chemistry there, retiring in 1921 after thirty-one years of service in the College (now the Military College of Science). Most of Hodgkinson's work was thus carried out at Woolwich, and much of it was directed to Service problems and to training many generations of gunner officers, who look back to this 